\title{
La Interdisciplinariedad En El Aula De Educación Secundaria: Una Investigación A Través De La Opinión Del Profesorado De Las Áreas De Música, Lengua Castellana Y Literatura, Y Ciencias Sociales.
}

\section{Carmen M. Zavala Arnal}

Docente investigadora

Doctora en Ciencias de la Educación por la Universidad de Zaragoza, España

Conservatorio Profesional de Música de Huesca

\section{Jorge Ramón Salinas}

Docente investigador

Doctor en Historia del Arte por la Universidad de Zaragoza, España.

Instituto de Enseñanza Secundaria y

Bachillerato “Ramón y Cajal” de Huesca

doi: 10.19044/esj.2017.v13n19p281 URL:http://dx.doi.org/10.19044/esj.2017.v13n19p281

\begin{abstract}
This paper shows the results of a study which analyzes daily practice through the opinion of teachers in Spain, which prompt us to consider the need for interdisciplinary activities. The study focuses on three areas: Music, Spanish Language and Literature, and Social Sciences. The results point at a favourable opinion of teachers regarding interdisciplinarity between the three subjects, although they highlight the obstacles arising from the curricular and educational context. The aim is to contribute to the integration in learning/teaching processes of interdisciplinary activities which can favour teachers as well as learners of these subjects.
\end{abstract}

Keywords: Interdisciplinarity, Curriculum, Secondary Education, Teachers

\section{Resumen}

En el presente artículo se muestran los resultados de una investigación en la que se analiza la práctica diaria en el aula de Educación Secundaria Obligatoria en España a través de la opinión del profesorado, que nos lleva a considerar la necesidad de la realización de actividades de tipo interdisciplinar. El estudio se centra en tres áreas: Música, Lengua Castellana y Literatura, y Ciencias Sociales. Los resultados apuntan a una favorable opinión del profesorado respecto a las estrategias de la 
interdisciplinariedad entre las tres áreas, si bien se ponen en relieve los obstáculos procedentes del contexto curricular y educativo. De esta forma, se pretende contribuir a la integración en los procesos de enseñanza-aprendizaje de actividades de carácter interdisciplinar que puedan favorecer tanto a los docentes como a los estudiantes de las áreas implicadas.

Palabras Clave: Interdisciplinariedad, Currículum, Enseñanza Secundaria, Profesorado

\section{Introducción:}

En las últimas décadas, se ha pretendido unificar en el ámbito docente el conocimiento de diferentes materias a través de las estrategias de la interdisciplinariedad. Las iniciativas van desde la puesta en marcha de estructuras académicas y curriculares más flexibles que conduzcan al currículum integrado (Torres, 2001), hasta la interrelación epistemológica entre distintas áreas de conocimiento (Pedroza Flores, 2006), siempre en aras de favorecer el bienestar educativo de la comunidad implicada (Zabala, 1999).

Respecto al marco teórico que sostiene el concepto de interdisciplinariedad, este es muy amplio y ocupa un plano destacado en el mundo de la educación (Piaget, 1979; Bunge, 2001), especialmente en el ámbito universitario y superior (Apostel, 1979; Palmade, 1983; Cobo Suero, 1986; Pedroza Flores, 2006). En la Enseñanza Secundaria ha sido objeto de algunos estudios, desde el análisis del currículo (Castañer, Trigo, 1995; Sáenz, 1997; Torres, 2001) hasta la propuesta de realización de actividades específicas (Zavala, Ramón, 2016). Todos los autores coinciden en que la interdisciplinariedad es uno de los conceptos recurrentes en el lenguaje educativo desde hace algunas décadas, y en que se muestra en la actualidad de forma perenne a todo cambio legislativo y formal. Una aproximación a su concepto nos explica cómo la interdisciplinariedad consiste en la coordinación, prevista de antemano, de dos o más disciplinas para estudiar un área o concepto determinado. Supone, por tanto, la interacción de estas disciplinas a través de diversos canales de comunicación. Estas pueden ir desde el simple intercambio de ideas hasta la integración mutua de leyes, teorías, hechos, conceptos, habilidades, hábitos, normas de conducta, sentimientos, valores a desarrollar, metodologías, formas de organización de las actividades y de las investigaciones (Apostel, 1983).

Desde la posición del profesorado, donde se contextualiza nuestro estudio, la interdisciplinariedad corresponde a una concepción de carácter socio-crítico que promueve el aprendizaje global (Delors, 1996), y que busca el desarrollo del pensamiento complejo (Morin, 2006) y la práctica reflexiva (Perrenoud, 2004). Respecto a la opinión del profesorado sobre las 
estrategias de la interdisciplinariedad, existen algunos estudios dedicados al punto de vista de los docentes. Castañer Balcells y Trigo Aza (1995), a través de una experiencia de investigación-acción que parte de un seminario de debate entre docentes de un centro educativo, constatan la evolución hacia un modelo didáctico de tipo interdisciplinar que permite la interrelación de algunas áreas del currículo. Por su parte, Altava Rubio, Pérez Serrano y Ríos García (1999) ponen en relieve que la formación del profesorado en estas cuestiones ha repercutido positivamente en la actitud diaria del aula, si bien no lo reflejan de manera cuantitativa. Chacón, Chacón y Alcedo (2012) señalan en su estudio, protagonizado por tres profesores de distintas áreas, la necesidad de promover la iniciativa docente a través del trabajo cooperativo. En los resultados muestran cómo los proyectos de tipo interdisciplinar favorecen la construcción del conocimiento y el grado de desarrollo de la conciencia de la formación permanente.

A efectos prácticos, sin embargo, se pueden constatar año tras año las escasas relaciones reales que se establecen en el proceso de enseñanzaaprendizaje entre algunas áreas de conocimiento. Estas "no relaciones" puede observarse particularmente entre las áreas de Música, Lengua Castellana y Literatura, y Ciencias Sociales, de Educación Secundaria, en la que ambientamos nuestro estudio, a pesar de que en el currículum vigente en España se hace referencia a algunos de sus ámbitos de interacción a través de los contenidos, los criterios, las competencias y los estándares de aprendizaje. Esto parece deberse a una falta de tradición en el aula de Educación Secundaria en el trabajo interdisciplinar, en contra de las tendencias científicas y sociales actuales. Mientras que en los medios de comunicación, así como en otros contextos educativos, estas conexiones son cada vez más valoradas, la enseñanza formal en la Educación Secundaria tiende a desaprovecharlas o a ignorarlas (Torres, 2001; Morin, 2006). En este sentido, se pierde una excelente oportunidad de renovación y avance en la introducción de nuevas estrategias didácticas en las áreas citadas. Por ello, se intenta proponer desde esta investigación algunas vías de reflexión en aras de una mejora de la situación.

En este sentido, se presenta este estudio que se desarrolla en tres centros educativos de la provincia de Huesca, en la Comunidad Autónoma de Aragón (España), donde se imparte Educación Secundaria y Bachillerato, y se centra en las opiniones del profesorado que imparte docencia en el $3^{\circ}$ curso de Educación Secundaria en las siguientes áreas:

- $\quad$ Música.

- $\quad$ Lengua Castellana y Literatura.

- Ciencias Sociales.

Para ello se recogen las opiniones de un grupo de profesores y profesoras especialistas respecto a diversas cuestiones relacionadas con la 
interdisciplinariedad en el ámbito educativo. La hipótesis plantea, desde un reconocimiento amplio de su utilidad, que el profesorado no lleva a la práctica suficientemente esta estrategia en su labor docente diaria.

Para la realización de la investigación, se parte de unos objetivos, que resumimos en los siguientes:

- Analizar el pensamiento y las opiniones del profesorado implicado sobre las relaciones entre las disciplinas y las causas de la falta de trabajo interdisciplinar.

- Obtener ciertas conclusiones que nos permitan detectar las causas, y revelar los ámbitos de interacción entre las disciplinas.

- Propiciar en el futuro el desarrollo de actividades de tipo interdisciplinar que favorezcan los procesos de enseñanza-aprendizaje de las tres áreas.

La enseñanza ha de convertirse en un proceso que tenga en cuenta la diversidad de la realidad objetiva. Esto exige un trabajo metodológico que implique la labor del profesorado de Educación Secundaria a través de su compromiso con la puesta en marcha de las estrategias de la educación.

\section{Metodología:}

\section{Participantes e instrumento de investigación}

Se trata de una investigación de tipo cuantitativo y cualitativo cuyo instrumento de investigación principal es el cuestionario. El estudio se ha realizado, por tanto, atendiendo a la elaboración del mismo, compuesto por 22 ítems de preguntas cerradas y 1 ítem de pregunta abierta cerradas, y a la recogida de datos.

El grupo de participantes está constituido por un total de veinte profesoras y profesores de Educación Secundaria.

\begin{tabular}{|l|l|l|}
\hline & MUJERES & HOMBRES \\
\hline SEXO & 13 & 7 \\
\hline EDAD & & \\
\hline Menos de 30 años & & \\
\hline Entre 30 y 50 años & 8 & 2 \\
\hline Más de 50 años & 8 & 2 \\
\hline TITULACIÓN & & \\
\hline Diplomado & 13 & \\
\hline Licenciado & & 6 \\
\hline Doctor & & 1 \\
\hline EXPERIENCIA DOCENTE & 1 & \\
\hline Menos de 10 años & 5 & 1 \\
\hline Entre 10 y 20 años & 9 & 3 \\
\hline Más de 20 años & & 3 \\
\hline ÁREAS IMPARTIDAS & 4 & \\
\hline Música & 4 & 3 \\
\hline Lengua y Literatura & 4 & 4 \\
\hline Historia/Geografía & 1 \\
\hline
\end{tabular}

Tabla 1. Características del grupo de participantes. 
Para la investigación, se ha diseñado un cuestionario original formado por 23 ítems o cuestiones, 22 de carácter cerrado y 1 de carácter abierto, cuyas respuestas nos permiten obtener unos resultados determinados, y en base a estas, unas conclusiones finales en relación a la confirmación o falsación de la hipótesis presentada. De esta forma, el paradigma cuantitativo nos brinda una información desde una perspectiva estable y realista a través de las mediciones cuantitativas que nos proporcionan los cuestionarios, realizados por docentes de Educación Secundaria de las áreas de Música, Lengua Castellana y Literatura, y Ciencias Sociales.

Las dimensiones establecidas, por agrupamiento de ítems, son cinco:

- Interdisciplinariedad(concepto, posibilidades): ítems 2/3/4/5/6/7/8//22

- Profesorado: ítems $1 / 9 / 10 / 18 / 19 / 20$

- Currículo: ítems 11/12

- Equipos directivos: ítems 13/14

- Alumnado: ítems 15/16/17/21

De los 23 ítems, 22 recogen las opiniones de los encuestadas/os en una escala que va desde el "muy en desacuerdo", "en desacuerdo", "ni de acuerdo ni en desacuerdo", "de acuerdo", y "muy de acuerdo. A las respuestas se les ha asignado una valoración que va desde el 1 (muy en desacuerdo) al 5 (muy de acuerdo). El último ítem -23- es una pregunta abierta a través de la cual se indaga acerca de la opinión del profesorado sobre temas que se podrían trabajar desde la interdisciplinariedad entre las áreas.

A continuación, se reproducen los ítems del cuestionario:

ÍTEM 1: "El currículo integrado, la interdisciplinariedad, los equipos docentes, etcétera, se han convertido en tópicos alejados y con poca presencia efectiva en la práctica docente”.

ÍTEM 2: "La interdisciplinariedad expresa la correlación entre las diversas disciplinas -reconocidas como independientes- pero relacionadas con conexiones que posibilitan un cierto grado de vinculación”.

ÍTEM 3: "La interdisciplinariedad dignifica el trabajo docente, crea el escenario y las condiciones para la elaboración conjunta de los saberes”.

ÍTEM 4: "Música, Lengua y Literatura, Ciencias Sociales, son áreas curriculares que pueden poner de relieve -con claridad- la conexión entre los conocimientos que imparten. Entre ellos, destacamos el conocimiento y valoración del patrimonio material e inmaterial”.

ÍTEM 5: "Lengua Castellana y Literatura, Ciencias Sociales, Música. ¿Le parece adecuado este orden de importancia desde la perspectiva de las exigencias de la sociedad?”.

ÍTEM 6: "Desde el punto de vista de la formación integral del alumno/a formado tanto en conocimientos como en valores de sensibilidad, crítica 
reflexiva, colaboración, solidaridad- ¿Estaría de acuerdo con la ordenación citada?”.

ÍTEM 7: “Ciencias Sociales y Música: la Música es fuente de la Historia y, a la vez, objeto de la Historia. La música es una actividad fundamental del ser humano con un importante papel en la sociedad. Por otro lado, el patrimonio histórico-artístico nos brinda diversas muestras de iconografía musical. Estas relaciones no deben ser desaprovechadas por la enseñanza”.

ÍTEM 8: "Música, y Lengua Castellana y Literatura. La música es un lenguaje y comparte con la palabra ritmo, entonación, fonética, pausas, cadencias. Por otro lado existen importantes conexiones entre la obra literaria y la obra musical. La práctica docente debe sacar partido a esta cuestión”.

ÍTEM 9: "Es un hecho que los profesores/as de las áreas de Música, Lengua Castellana y Literatura, y Ciencias Sociales, establecen pocas conexiones entre ellas".

ÍTEM 10: "La principal razón para esta desconexión radica en la poca tradición del trabajo interdisciplinar en los centros educativos de Educación Secundaria”.

ÍTEM 11: "Una posible razón que dificulta el trabajo interdisciplinar la encontramos en el currículo de las distintas materias con rupturas, saltos y desfases entre sus contenidos".

ÍTEM 12: "El profesorado, en su práctica diaria, tiende a subsanar y corregir los desfases y/o lagunas que aparecen en el currículo. ¿Vería apropiado un análisis -en común- del currículo para conseguir evitar estos inconvenientes?".

ÍTEM 13: "En los documentos de centro se recogen la bondades y la adecuación del trabajo interdisciplinar. En los centros educativos existe la suficiente flexibilidad de tiempos y espacios que facilitan esta modalidad del trabajo docente".

ÍTEM 14: "El profesorado se ve animado a realizar proyectos de interdisciplinariedad educativa desde los órganos directivos, pero luego no encuentra el apoyo necesario porque hay un rendimiento académico que debe cumplirse".

ÍTEM 15: "Los obstáculos que presenta la sociedad actual se refleja en el alumnado, que dificultan el trabajo docente y la motivación e ilusión que generan las nuevas estrategias educativas".

ÍTEM 16: "La heterogeneidad del alumnado, las actitudes negativas, las crisis de valores son impedimentos sustanciales para cualquier intento de puesta en marcha de nuevas estrategias de interdisciplinariedad".

ÍTEM 17: "Las opiniones y prejuicios del alumnado -por presiones del entorno familiar, amistades, medios de comunicación- condicionan 
negativamente e imposibilitan un cambio de actitud ante las nuevas estrategias educativas”.

ÍTEM 18: "El profesorado de estas materias, así como el profesorado en general, trabaja con interés y cumple la normativa pero obvia introducir estrategias de trabajo interdisciplinar”.

ÍTEM 19: "La fuerte individualidad de cada profesor, que puede llegar a obstaculizar la aceptación de la opinión del otro, dificulta el trabajo interdisciplinar en los centros educativos".

ÍTEM 20: "La incomprensión de los compañeros -que se desplaza desde la indiferencia a la oposición- desanima a las personas que intentan optimizar su labor diaria en el aula”.

ÍTEM 21: "La falta de reconocimiento social -desde la autoridad educativa a la sociedad- del trabajo de los docentes, ahoga muchas de sus iniciativas educativas".

ÍTEM 22: "La interdisciplinariedad se encuentra en todas las tendencias científicas y sociales, y sus conexiones son cada vez más valoradas. Los docentes no pueden dar la espalda a esta realidad".

ÍTEM 23: “¿Qué posibles temas y ámbitos de interacción (curriculares, materiales, procedimentales, etcétera) cree que se podrían trabajar desde la interdisciplinariedad entre estas áreas?”.

\section{Procedimiento}

Se ha partido del método cuantitativo tomando el cuestionario como herramienta para el análisis de datos. Para contabilizar los registros que este nos proporciona, se ha utilizado la medición nominal, que expresa a través de los porcentajes obtenidos la presencia de variables dentro de cada ítem (Ferrando, 1992). Las fases que se han seguido para la elaboración de los cuestionarios son las siguientes :

- Determinación de los objetivos principales de estudio e hipótesis, que constituyen el cimiento de los cuestionarios.

- Selección de un grupo de profesores de las tres áreas de conocimiento implicadas en tres centros educativos diferentes. Envío de información sobre la naturaleza, los objetivos y las características del estudio.

- Diseño y elaboración de un cuestionario original formado por 22 ítems en los que los docentes deben expresar numéricamente su grado de acuerdo o desacuerdo en relación a varias cuestiones estrechamente ligadas con las interdisciplinariedad entre sus áreas de conocimiento. A su vez, estos ítems han sido agrupados en cinco dimensiones referidas a las cuestiones que se ven afectadas: Interdisciplinariedad (concepto y posibilidades), profesorado, currículo, equipos directivos, y alumnado. Al cuestionario se añade un ítem más, el número 23, de carácter abierto, para que los docentes expresen por 
escrito propuestas concretas respecto a los contenidos curriculares en los que se pueden interrelacionar las materias.

- Realización y envío de los cuestionarios por parte de los docentes implicados, y análisis de los mismos.

\section{Análisis de datos y resultados:}

Los datos que se obtienen de los cuestionarios nos proporcionan unos resultados que nos suministran el conocimiento de un fenómeno real respecto a nuestro objeto de estudio.

A continuación, se muestran los resultados a través de un gráfico que recoge los porcentajes de respuesta obtenidos a través de la asignación de una valoración (series), que van desde el 1, "muy en desacuerdo" al 5 "muy de acuerdo"), a cada uno de los 22 ítems de preguntas cerradas.

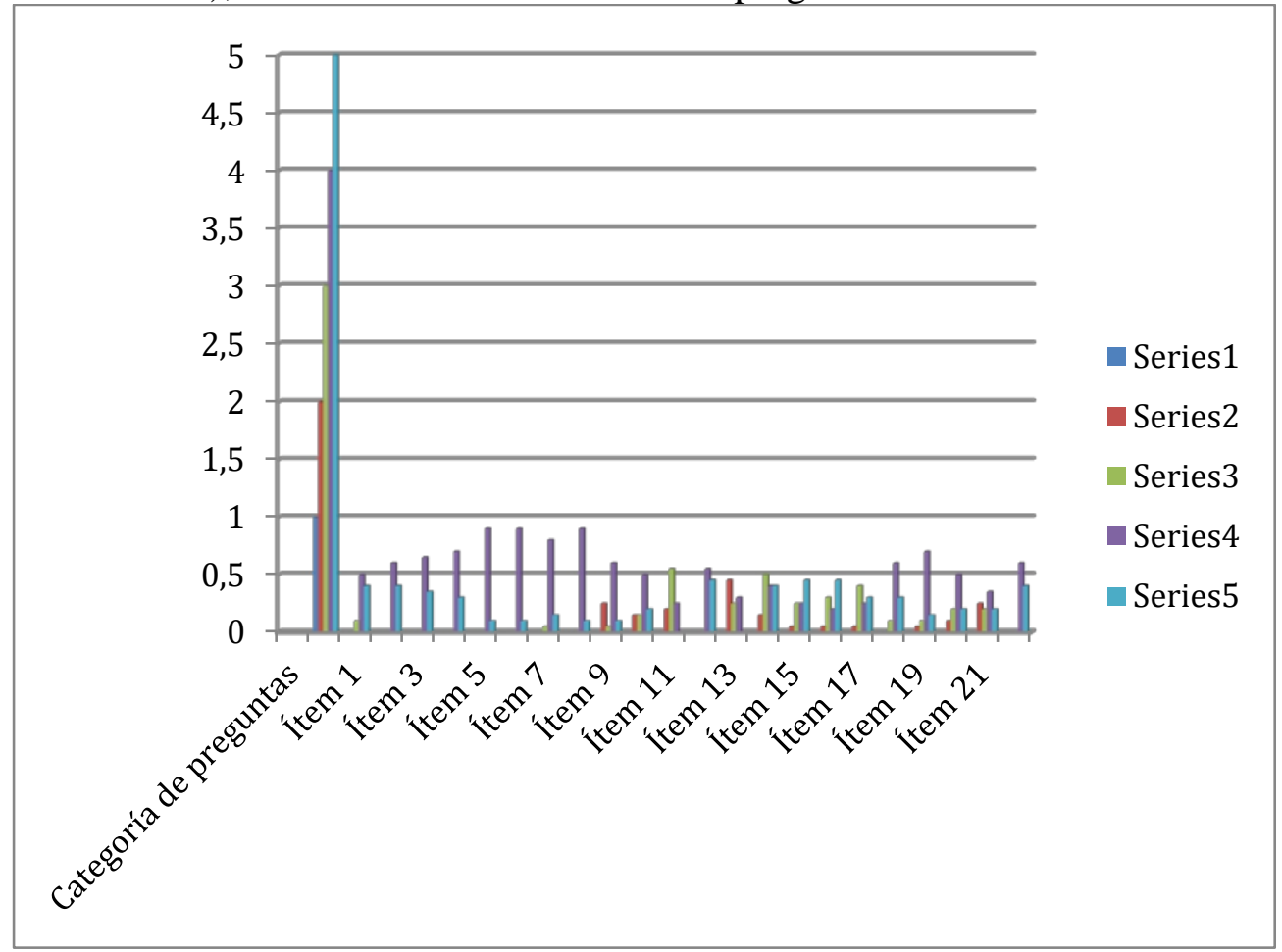

Tabla 2. Resultados del cuestionario.

Un aspecto a resaltar en los resultados del ítem 23, de respuestas abiertas y que por tanto no se refleja en la tabla, son los ámbitos y contenidos propuestos por los profesores/as en los que interaccionen las disciplinas señaladas, que han sido los siguientes:

- Los períodos histórico-estéticos.

- La cronología histórica.

- El patrimonio histórico-artístico y musical como reflejo de una época. 
- La iconografía musical.

- Los temas literarios en las artes y la música.

- Los estilos artísticos, musicales y literarios, y su relación en el espacio (geografía) y el tiempo (historia).

- La historia y el pensamiento.

- El lenguaje musical y el lenguaje verbal.

- Los espacios físicos y virtuales de interacción: el museo físico, el museo virtual, las TIC, etcétera.

\section{Conclusion:}

A través del estudio que aquí se presenta, basado en la opinión del profesorado respecto a las estrategias de la interdisciplinariedad en la Educación Secundaria, se ha podido contribuir a reflexionar sobre la realidad en el aula, la posición y actitud del profesorado, y el papel que juega el contexto curricular y educativo (equipos directivos y alumnado) en aras de mejorar estos procesos.

Por otro lado, se ha podio confirmar la hipótesis inicial de este estudio, que señalaba a la escasez, a efectos prácticos, de relaciones en los procesos de enseñanza-aprendizaje.

La conclusiones, por otro lado, muestran algunos ámbitos de interacción que puedan propiciar en el futuro la puesta en marcha de actividades de tipo disciplinar entre las tres áreas implicadas, que acabamos de señalar. De esta forma, se han alcanzado los objetivos propuestos en este estudio.

Respecto a los resultados específicos que se desprenden tras el análisis pormenorizado de cada ítem del cuestionario, se confirma la opinión favorable del profesorado hacia la práctica de actividades de tipo interdisciplinar entre las tres áreas, poniendo de relieve que las dificultades se sustancian, en cierto modo, en el currículo de las áreas implicadas y, más específicamente, en la falta de disposición y flexibilidad por parte del entorno, especialmente del centro educativo.

Otro aspecto esencial señala su puesta en práctica en la labor diaria del aula, que resulta un poco más compleja de dilucidar. La implicación del profesorado no se admite claramente en las opiniones vertidas, y se tiende a evitar posturas firmes que coloquen la actitud del mismo como condicionante en la toma de conciencia y traslado en la práctica de las estrategias educativas de la interdisciplinariedad. Respecto al alumnado, éste tampoco se ve como elemento decisivo.

Sin embargo, como ya hemos señalado, existen dos dimensiones que sí resultan clarificadoras en relación a la interdisciplinariedad que, por orden de importancia, son el currículo y los órganos directivos del centro. Se reconoce la imperfección y deficiencias en los currículos -desfases, saltos, omisionesy la necesidad de un análisis conjunto del mismo que elimine estos 
obstáculos. Esto exige, a su vez, un cambio de actitud en los docentes que les lleve a un trabajo en equipo, cooperativo y de colaboración, entre los departamentos didácticos.

Los resultados, por tanto, arrojan nuevas vías que se concretan en contenidos específicos en los que se interrelacionan las áreas propuestas, lo que muestra la posibilidad de encaminarse hacia un modelo didáctico interdisciplinar, tal y como apuntan Castañer Balcells y Trigo Aza (1995). Por otro lado, los resultados apuntan a una buena consideración por parte del profesorado a participar y mejorar las estrategias y el papel de los factores que intervienen en la integración del currículo, tal y como se recoge en otro estudio (Chacón, Chacón y Alcedo, 2012).

Podemos concluir que la realización y el análisis de los resultados da como fruto una nueva perspectiva acerca de la situación actual del trabajo interdisciplinar en el aula de Educación Secundaria en España. Desde aquí, se pretende contribuir en el futuro al diseño de nuevas propuestas curriculares y a la puesta en práctica de actividades de carácter interdisciplinar que redunden en el enriquecimiento de los procesos de enseñanza-aprendizaje de las áreas señaladas. Queda pendiente la realización de estudios desde el punto de vista del alumnado, así como desde otras áreas en las que se puedan establecer relaciones de interdisciplinariedad. Por otro lado, se podrían establecer resultados y conclusiones más precisos con una muestra mayor de participantes a través de la comparación con estudios similares.

\section{References:}

1. Altava Rubio, V., Pérez Serrano, I. C. y Ríos García, I. M (1999). La interdisciplinariedad como instrumento de formación del profesorado. Revista electrónica interuniversitaria de formación del profesorado, 2 (1), 241-250.

2. Apostel, L. (1983). Las ciencias humanas: muestra de relaciones interdisciplinarias. En Tom Bottomore (Ed.), Interdisciplinariedad y ciencias humanas (pp. 71-164). Madrid: Tecnos.

3. Bunge, M. (2001). Construyendo puentes entre las ciencias sociales. En Octavio Bunge, Anthony Giddens, Robert Castel, Alain Touraine (Eds.), Desigualdad y Globalización. Cinco conferencias (pp. 47-74). Buenos Aires (Argentina): Universidad de Buenos Aires.

4. Castañer Balcells, M., Trigo Aza, E. (1995). Interdisciplinariedad en la Educación Secundaria. Barcelona: Editorial Inde.

5. Cabo Hernández, J. M., Granda Vera, J. y Sebastián Sánchez, F. (1990). Interdisciplinariedad y globalización en la formación inicial del profesorado. Un caso de investigación-acción. Revista interuniversitaria de formación del profesorado, 9, 15-42. 
6. Chacón Corzo, M. A., Chacón, C. T. y Alcedo Y. A. (2012). Los proyectos de aprendizaje interdisciplinarios en la formación docente. Revista mexicana de investigación educativa, 17 (54), 877-902.

7. Cobo Suero, J. M. (1986). Interdisciplinariedad y Universidad. Madrid: Universidad Pontificia de Comillas.

8. Delors, J. (1996). La educación encierra un tesoro. Madrid: Santillana, ed. Unesco.

9. Ferrando, M. (1992). La Encuesta. El análisis de la realidad social: métodos y técnicas de investigación. Madrid: Alianza.

10. Morin, E. (2006). La mente bien ordenada: repensar la reforma, reformar el pensamiento. Barcelona: Seix Barral.

11. Palmade, G. (1979). Interdisciplinariedad e ideologías. Madrid: Narcea.

12. Pedroza Flores, R. (2006). La interdisciplinariedad en la Universidad. Tiempo de educar, 7 (13), 69-98.

13. Perrenoud, P. (2004). Desarrollar la práctica reflexiva. Barcelona: Graó.

14. Piaget, J. (1979). La epistemología de las relaciones interdisciplinarias. Mecanismos del desarrollo mental. Madrid: Ed. Nacional de Madrid.

15. Sáenz Barrio, O. (1997). La formación didáctica de los profesores de Educación Secundaria. Revista interuniversitaria de formación del profesorado, 28, 39-51.

16. Torres, J. (2001). Globalización e interdisciplinariedad: el currículum integrado. Madrid: Editorial Morata.

17. Zabala, A. (1999). Enfoque globalizador y pensamiento complejo. Barcelona: Grao.

18. Zavala Arnal, C. M., Ramón Salinas, J. (2016). El museo como espacio interdisciplinar. Una reflexión acerca de la contribución de la iconografía musical en la didáctica del patrimonio en la Educación Secundaria. En Laura Arias, Ana Isabel Ponce Gea y David Verdú (Eds.), Estrategias y recursos para la integración del patrimonio y los museos en la educación formal (pp. 89-94). Murcia: Universidad de Murcia. 